\title{
III-V/Silicon Photonics for Short-Wave Infrared Spectroscopy
}

\author{
Gunther Roelkens, William M. J. Green, Member, IEEE, Bart Kuyken, Xiaoping Liu, Nannicha Hattasan, \\ Alban Gassenq, Laurent Cerutti, Jean Baptiste Rodriguez, Richard M. Osgood, Fellow, IEEE, \\ Eric Tournié, and Roel G. Baets, Fellow, IEEE
}

(Invited Paper)

\begin{abstract}
In this paper, we review our work on III-V/SOI photonic integrated circuits for short-wave infrared applications. We focus on the integration of short-wave infrared photodetectors on a silicon photonics platform and on the generation of a short-wave infrared supercontinuum using the $\chi^{(3)}$ nonlinearity in silicon photonic wires. In addition, the performance of a silicon optical parametric amplifier is reviewed, as a first step towards constructing an integrated tunable short-wave infrared parametric oscillator.
\end{abstract}

Index Terms-Heterogeneous integration, short-wave infrared, silicon photonics.

\section{INTRODUCTION}

$\mathbf{T}$ HE SILICON photonics platform is broadly being studied as an attractive platform for the extremely dense integration of optical functions at telecommunication wavelengths [1]. The use of CMOS fabrication technology, leading to high yield and the potential for co-integration of electronic circuits, and the high refractive index contrast available on a siliconon-insulator waveguide platform $(\Delta \mathrm{n} \cong 2)$ are the main drivers for this evolution. While photonic integrated circuits were primarily intended to serve the telecommunication and datacommunication markets, the use of silicon photonics in other applications is also being explored, predominantly geared towards sensing. Examples of such fields of research

Manuscript received September 1, 2011; revised November 2, 2011; accepted November 18, 2011. Date of current version January 24, 2012. This work was supported in part by the Flemish IWTSBO Glucosens Project, the FP7-ERC-MIRACLE Project, and the FP7-ERC-InSpectra. This work was carried out in part by the framework of the IWT-SBO-Glucosens Project, the FP7-ERC-InSpectra Project, and the FP7-ERC-MIRACLE Project.

G. Roelkens, B. Kuyken, N. Hattasan, A. Gassenq, and R. G. Baets are with the Photonics Research Group, Ghent University/Illinois Manufacturing Extension Center, Ghent 9000, Belgium. They are also with the Center for Nano- and Biophotonics, Ghent University, Ghent 9000, Belgium (e-mail: gunther.roelkens@intec.ugent.be; bart.kuyken@intec.ugent.be; nannicha.hattasan@intec.ugent.be; alban.gassenq@intec.ugent.be; roel.baets@in tec.ugent.be).

W. M. J. Green is with IBM Thomas J. Watson Research Laboratory, Yorktown Heights, NY 10598 USA (e-mail: wgreen@us.ibm.com).

X. Liu is with the OFS Laboratories, Somerset, Murray Hill, NJ 07974-0636 USA (e-mail: xpliu.columbia@gmail.com).

L. Cerutti, J. B. Rodriguez, and E. Tournié are with nanoMIR, Université Montpellier 2, Montpellier 34095, France (e-mail: cerutti@univ-montp2.fr; rodriguez@ies.univ-montp2.fr; etournie@univ-montp2.fr).

R. M. Osgood is with the Department of Electrical Engineering, Columbia University, New York, NY 10027 USA (e-mail: osgood@columbia.edu).

Color versions of one or more of the figures in this paper are available online at http://ieeexplore.ieee.org.

Digital Object Identifier 10.1109/JQE.2011.2178090 are silicon-based bio-sensors [2], gas sensors [3] and the development of bio-medical instrumentation [4]. In these applications, one typically retains the use of the telecommunication wavelength range, for reasons of experimental simplicity, the availability of characterized building blocks, etc.

However, a number of applications would benefit from the use of highly integrated silicon photonic circuits specifically tailored to operate at longer wavelengths. For the specific field of spectroscopic sensing, probing of the molecular fingerprint absorption lines of molecules exploits the strong absorption features of molecules in the short-wave $(1.4-3 \mu \mathrm{m})$ and midwave $(3-8 \mu \mathrm{m})$ infrared [5]. The availability of an integration platform covering these two wavelength ranges could enable ultra-compact, low-cost sensor solutions, designed to outperform existing solutions in terms of their selectivity, sensitivity, portability, and power consumption.

In this paper we report on our first steps towards the development of such a long-wavelength silicon photonics platform, by addressing the $2-2.5 \mu \mathrm{m}$ wavelength range. We will first focus our discussion to the heterogeneous integration of III-V short-wave infrared photodetectors on a silicon waveguide circuit as a milestone towards the realization of complete postdispersive spectrometer chips [6]. We then also report on the use of the strong $\chi^{(3)}$ nonlinearity in silicon photonic wires to generate short-wave infrared radiation, in the form of an onchip short-wave infrared supercontinuum or through on-chip optical parametric amplification and oscillation.

\section{Long Wavelength III-V / Silicon Photonics}

Depending on the particular wavelength range of operation, standard silicon-on-insulator waveguide structures can be used with a silicon waveguide core layer on top of a $\mathrm{SiO}_{2}$ cladding layer. While silicon is transparent to beyond $6 \mu \mathrm{m}$, the $\mathrm{SiO}_{2}$ cladding limits the wavelength range of operation of the SOI waveguide platform to about $4 \mu \mathrm{m}$ [7]. Alternative waveguide structures such as free-standing silicon membranes [8], silicon-on-sapphire [9], silicon-on-nitride [7], germanium-on-silicon [7] or silicon-on-porous silicon [10] need to be considered when wavelengths beyond $4 \mu \mathrm{m}$ are of interest. While in the short-wave infrared, standard SOI waveguide structures can be used (note that in this paper a $220 \mathrm{~nm}$ silicon waveguide layer on top a $2 \mu \mathrm{m}$ buried oxide layer was used), until recently the silicon waveguide platform has lacked light sources and photodetectors for this 


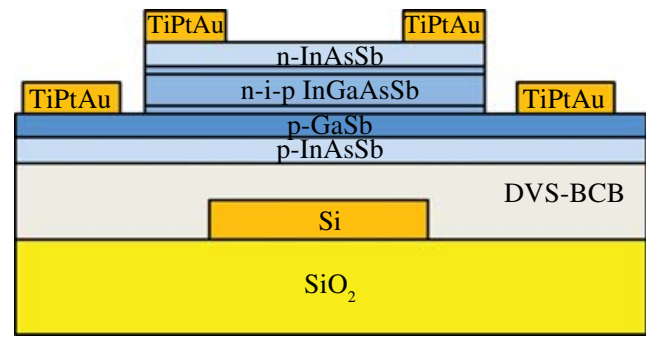

(a)

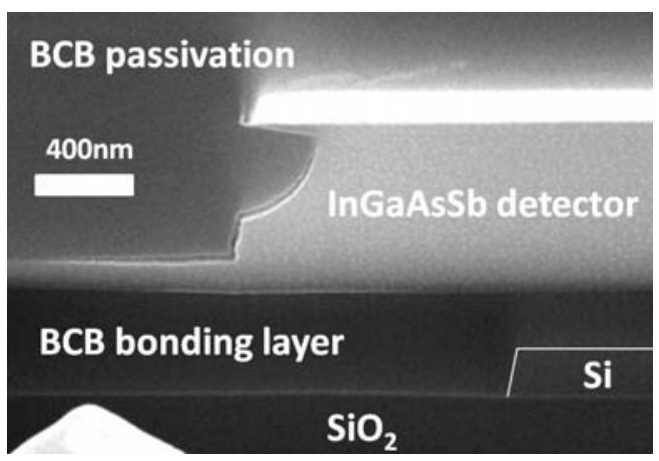

(b)

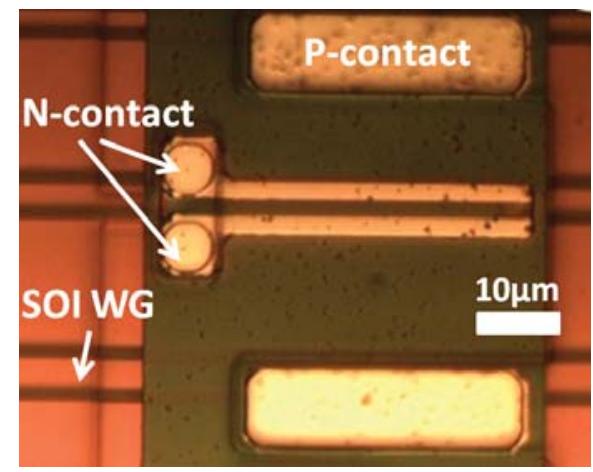

(c)

Fig. 1. (a) Schematic cross-section of the heterogeneous III-V/silicon photodetector. (b) SEM cross-section of the fabricated device. (c) Top-down microscope image of the InGaAsSb photodetector aligned to the underlying silicon waveguide circuit.

wavelength range. Therefore, a process for the integration of III-V semiconductors on top of the silicon waveguide circuits was developed, by means of epitaxial layer transfer with an adhesive bonding layer. This process was originally developed to transfer InP-based epitaxy to a silicon waveguide circuit for $1.3 \mu \mathrm{m}$ to $1.7 \mu \mathrm{m}$ applications. However, it is difficult to push the emission and/or absorption wavelength of InP/InGaAsP/InGaAlAs-based structures far beyond $2 \mu \mathrm{m}$ [11]. Moreover, an alternative semiconductor system, the InP-based quantum cascade materials, typically address wavelengths beyond $4 \mu \mathrm{m}$ [12]. Therefore, in this work a heterogeneous integration process for GaSb-based epitaxy was developed, which allows for band-to-band laser emission and photodetection in the $2-3.5 \mu \mathrm{m}$ wavelength range [13].

To illustrate the heterogeneous integration process, InGaAsSb-based photodetectors were integrated on a silicon waveguide circuit. The device layout is shown in Figure 1(a) [14]. The GaSb-based epitaxial layer stack, grown using

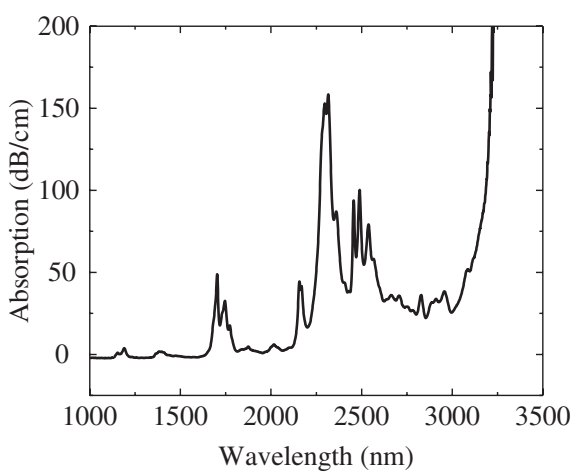

Fig. 2. Absorption spectrum of DVS-BCB in the short-wave infrared, measured using a photospectrometer setup.

molecular beam epitaxy on an n-type GaSb substrate, is bonded "upside down" onto the SOI waveguide wafer, using the same process as reported in [15], i.e. using a DVS-BCB adhesive bonding agent. After bonding, the GaSb growth substrate is removed by chemical wet etching with a mixture of $\mathrm{HF}, \mathrm{CrO}_{3}$ and water $(1: 1: 3 \mathrm{v} / \mathrm{v})$, using an InAsSb layer as an etch stop, which is in turn removed by a mixture of citric acid and hydrogen peroxide $(2: 1 \mathrm{v} / \mathrm{v})$, leaving a III-V epitaxial film attached to the silicon waveguide circuit, in which the III-V opto-electronic components can be defined. The epitaxial stack consists of $50 \mathrm{~nm}$ p-InAsSb $(1.0 \times$ $\left.10^{18} \mathrm{~cm}^{-3}\right), 50 \mathrm{~nm}$ p-doped $\left(1.0 \times 10^{18} \mathrm{~cm}^{-3}\right) \mathrm{GaSb}$ and a $50 \mathrm{~nm}$ p-doped $\left(1.0 \times 10^{18} \mathrm{~cm}^{-3}\right) \operatorname{In}_{0.21} \mathrm{Ga}_{0.79} \mathrm{As}_{0.19} \mathrm{Sb}_{0.81}$ layer as the p-zone of a p-i-n layer stack. An un-intentionally doped $500 \mathrm{~nm}$ thick $\operatorname{In}_{0.21} \mathrm{Ga}_{0.79} \mathrm{As}_{0.19} \mathrm{Sb}_{0.81}$ layer is used for the intrinsic absorbing region, and has a cut-off wavelength of $2.5 \mu \mathrm{m}$. The $\mathrm{n}$-type region consists of $\operatorname{In}_{0.21} \mathrm{Ga}_{0.79} \mathrm{As}_{0.19} \mathrm{Sb}_{0.81}$ and $50 \mathrm{~nm} \mathrm{InAs} \mathrm{s}_{0.91} \mathrm{Sb}_{0.09}$. Both are doped to $10^{18} \mathrm{~cm}^{-3}$. An In $\mathrm{As}_{0.91} \mathrm{Sb}_{0.09}$ layer is chosen as $\mathrm{n}$-type contact because of its lower bandgap $(0.35 \mathrm{eV})$ and low contact resistance.

The III-V mesa, $9 \mu \mathrm{m}$ wide, was defined using a combination of both dry $\left(\mathrm{CH}_{4}: \mathrm{H}_{2}\right)$ and wet etching to reduce the dark current of the photodetector. TiPtAu contacts were used both for n-type and p-type metallization. Figure 1(b) shows a SEM cross-section image of the integrated device while Figure 1(c) shows a top-down microscope image. The coupling from the silicon waveguide layer to the InGaAsSb photodetector is based on evanescent coupling between the SOI ( $3 \mu \mathrm{m}$ wide and $220 \mathrm{~nm}$ high silicon core) and III-V waveguide. While DVS-BCB is considered transparent in the telecommunication wavelength band, there are strong absorption bands in the short-wave infrared as shown in Figure 2. This implies that the DVS-BCB needs to be removed on top of the silicon waveguide circuits to avoid excessive waveguide losses, leaving the DVS-BCB only underneath the bonded III-V opto-electronic components. Currently a process is under development such that by applying a 5-10 nm aluminum oxide layer on the silicon waveguide circuit prior to spincoating of the DVS-BCB, this aluminum oxide can serve as an etch stop during the dry etch removal of the DVS-BCB bonding layer after III-V processing.

To characterize the short-wave infrared photodetectors, the light from a short-wave infrared tunable laser [16] was coupled 


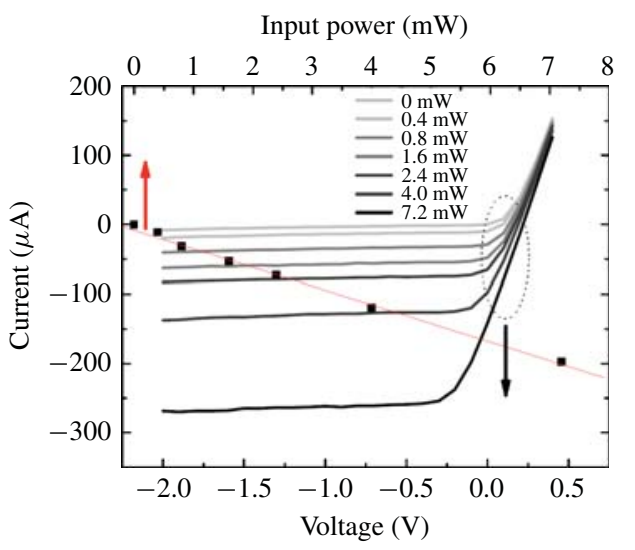

Fig. 3. I-V characteristics of the heterogeneously integrated short-wave infrared photodetector for different optical power levels in the silicon waveguide $(2.25 \mu \mathrm{m}$-TE polarization) and photocurrent at $0.1 \mathrm{~V}$ bias for different input power levels.

to a standard single mode fiber (SMF), which interfaces with the silicon waveguide circuit, after passing a polarization controller, using a one-dimensional grating coupler designed for the short-wave infrared. While a basic grating design with limited efficiency was used for the characterization of the devices, a proper design of such gratings allows for efficient $(-5 \mathrm{~dB}$ fiber-to-chip coupling efficiency experimentally obtained) and broadband (160 nm $3 \mathrm{~dB}$ bandwidth) coupling from a standard SMF to a single mode SOI waveguide [17]. The photodetector I-V curves at room temperature for various fiber-coupled power levels at $2.25 \mu \mathrm{m}$ are shown in Figure 3. Calibrating and then correcting for the fiber-chip coupling efficiency, an on-chip responsivity larger than $0.4 \mathrm{~A} / \mathrm{W}$ is obtained over a $200 \mathrm{~nm}$ wide band around $2.3 \mu \mathrm{m}$. The dark current at room temperature is $1.13 \mu \mathrm{A}(-0.1 \mathrm{~V}$ bias $)$. This leads to a Johnson-noise-limited noise equivalent power of $1.5 \mathrm{pW} / \mathrm{Hz}^{1 / 2}$. From Figure 1 a slight misalignment between the photodetector and the underlying silicon waveguide circuit can be observed, which impacts the responsivity. In future device runs therefore higher responsivity can be expected.

\section{Generation of ShORT-WaVe InfRared Radiation THROUGH NONLINEAR OPTICS}

In addition to photodetectors, a second component needed for a fully functional IR chip is a heterogeneously integrated short-wave infrared light source, mounted on the silicon waveguide platform. One possible approach would be to utilize the epitaxial layer transfer process described above to bond and lithographically define a GaSb-based semiconductor injection laser structure. Another valuable approach could make use of silicon nonlinear optical components specifically designed to generate broadband short-wave infrared radiation [18]-[21]. As will be shown in this section, the latter approach allows coverage of a much broader spectral range than what can be achieved with a single GaSb-laser epitaxial structure. The ultra-broadband characteristics of silicon nonlinear optical devices are very useful for spectroscopy applications. Of course, using nonlinear optics to generate broadband radiation still requires integration of a narrowband and bright optical

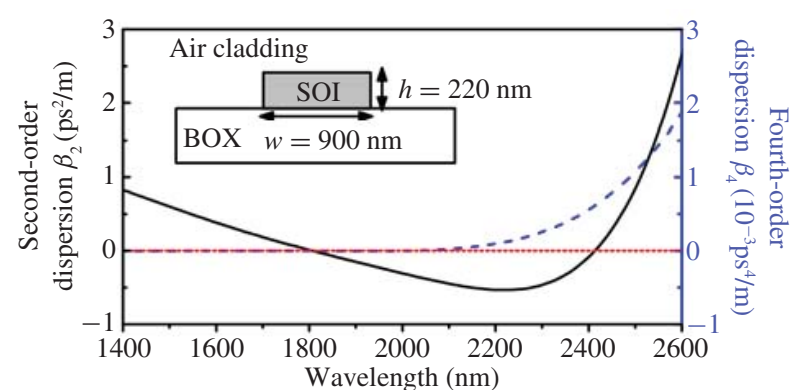

(a)

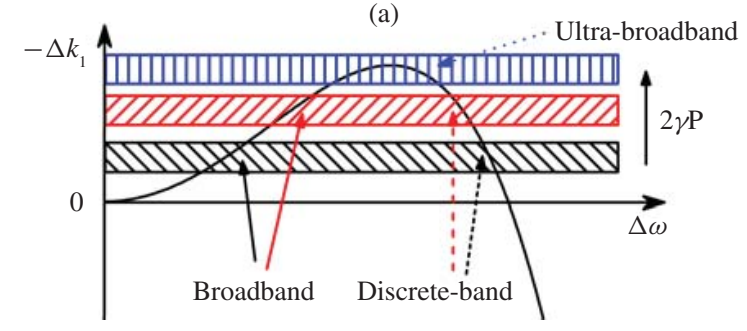

(b)

Fig. 4. (a) Second-order and fourth-order waveguide dispersion of the $900 \mathrm{~nm}$ $220 \mathrm{~nm}$ silicon photonic wire studied and graphical illustration of the phase matching condition. The curve shows the linear phase mismatch as a function of detuning from the pump. (b) Intersections with the horizontal boxes this linear phase mismatch is compensated by the nonlinear phase mismatch.

pump source on the chip, which could be realized using a heterogeneous InGaAsSb/SOI laser. In this section, we will focus on the basic nonlinear optical properties of silicon photonic wires in the short-wave infrared, after which we will demonstrate generation of a $1.5-2.5 \mu \mathrm{m}$ supercontinuum and wide-band optical parametric generation / amplification on a silicon chip using picosecond optical pump pulses.

Prior to this work, silicon photonics for nonlinear optics at telecommunication wavelengths had been explored in depth [22]-[28]. This interest stems from the large bulk $\chi^{(3)}$ optical nonlinearity of silicon combined with the tight confinement of the optical mode in a single-mode waveguide, along with the large range of waveguide dispersion engineering that is possible with a single-mode $\mathrm{Si}$ wire waveguide [29]-[31]. At telecommunication wavelengths, silicon however exhibits parasitic two-photon absorption, which limits the efficiency of the nonlinear processes at these wavelengths. Given the $1.12 \mathrm{eV}$ bandgap of silicon, this two-photon absorption process disappears above $2.2 \mu \mathrm{m}$, resulting in much higher efficiency nonlinear processes in the short-wave infrared [32]. For our nonlinear optics experiments here, $900 \mathrm{~nm}$ wide and $220 \mathrm{~nm}$ thick silicon strip wire waveguides are used with an air top cladding. The waveguide cross-section is shown in the inset of Figure 4(a). Using a cut back technique - by comparing the insertion loss of a $1 \mathrm{~cm}, 2 \mathrm{~cm}, 4 \mathrm{~cm}$ and $7 \mathrm{~cm}$ long spiral waveguides - the TE mode waveguide propagation loss is found to be approximately $2.5 \mathrm{~dB} / \mathrm{cm}$ across the 2050 $2450 \mathrm{~nm}$ wavelength range. Since the short-wave infrared wavelength generation relies upon phase matching of the interacting waves, waveguide dispersion engineering is of paramount importance. Indeed, efficient four-wave mixing (FWM) occurs only when

$$
\Delta k=\Delta k_{\text {lin }}+\Delta k_{\text {nonlin }}=k_{s}+k_{i}-2 k_{\text {pump }}+2 \gamma P=0
$$


in which $k_{\text {pump }}, k_{s}$ and $k_{i}$ are the linear propagation constants of the pump, signal and idler waves, respectively. The term $2 \gamma P$, in which $\gamma$ is the effective nonlinear parameter of the waveguide and $P$ is the peak power of the pump pulse, accounts for the self-phase and cross-phase modulation of the interacting waves [31]. Using a Taylor expansion of the waveguide dispersion relation around $\omega_{\text {pump }}$, and taking into account the conservation of energy in the FWM process, produces the phase matching condition

$$
\beta_{2} \Delta \omega^{2}+\frac{1}{12} \beta_{4} \Delta \omega^{4}+2 \gamma P=0
$$

in which $\Delta \omega$ is the frequency detuning between pump and signal (and also between pump and idler). The second- $\left(\beta_{2}\right)$ and fourth-order $\left(\beta_{4}\right)$ waveguide dispersion of the $900 \mathrm{~nm}$ wide photonic wire are plotted in Figure 4(a). Anomalous dispersion $\left(\beta_{2}<0\right)$ is obtained in the $1800-2400 \mathrm{~nm}$ wavelength range, while $\beta_{4}$ is small but positive. The effective nonlinear parameter of this waveguide is calculated to be $130-150(\mathrm{Wm})^{-1}$ at short-wave infrared wavelengths.

The phase matching condition described by equations (1) and (2) is graphically illustrated in Figure 4(b). Given the opposite signs of $\beta_{2}$ and $\beta_{4}$ exhibited by the $900 \mathrm{~nm} \times$ $220 \mathrm{~nm}$ waveguide design, Figure 4(b) shows that two phasematched frequency bands can be obtained around two detuning frequencies. These bands are labeled as the broad and the discrete band, respectively. At these detunings the linear and nonlinear phase mismatch compensate each other. Due to the steepness of the linear phase mismatch curve far away from the pump, the bandwidth of the discrete band is smaller than that of the broad band close to the pump. Increasing the pump power eventually leads to the merging of both bands, at which point ultra-broadband phase-matching is obtained. This effect was exploited in a recent experiment, in which optical parametric generation over a $580 \mathrm{~nm}$-wide wavelength band was demonstrated by sending a picosecond pulse train at $2.173 \mu \mathrm{m}$ wavelength with $13.5 \mathrm{~W}$ coupled peak input power through a 2-cm-long silicon photonic wire [33]. The optical parametric generation, shown in Figure 5(a), originates primarily from modulation instability, the amplification of background noise through ultra-broadband FWM with the pump. The intense modulation instability spectrum correlates with unprecedented values of on-chip parametric gain, exceeding $40 \mathrm{~dB}$ (Figure 5(c)). Moreover, we have demonstrated that on-chip gain can exceed $50 \mathrm{~dB}$ in narrow Raman-scatteringassisted bands, also shown in Figure 5(c). This gain spectrum was obtained by coupling a weak co-polarized continuous wave probe signal along with the pump pulse train, leading to the parametric amplification of the signal when the pump and signal overlap in time (hence the pedestal around the spiked continuous wave tones in Figure 5(b) on the red side of the pump) and the simultaneous generation of an idler pulse train. Taking into account the duty cycle of the pulse train ( 2 ps long pulses, $76 \mathrm{MHz}$ repetition rate) the effective amplification and conversion gain can be calculated as shown in Figure 5(c).

In order to further understand the processes involved in the generation of this short-wave infrared radiation, the generation of a supercontinuum was studied at different pump power

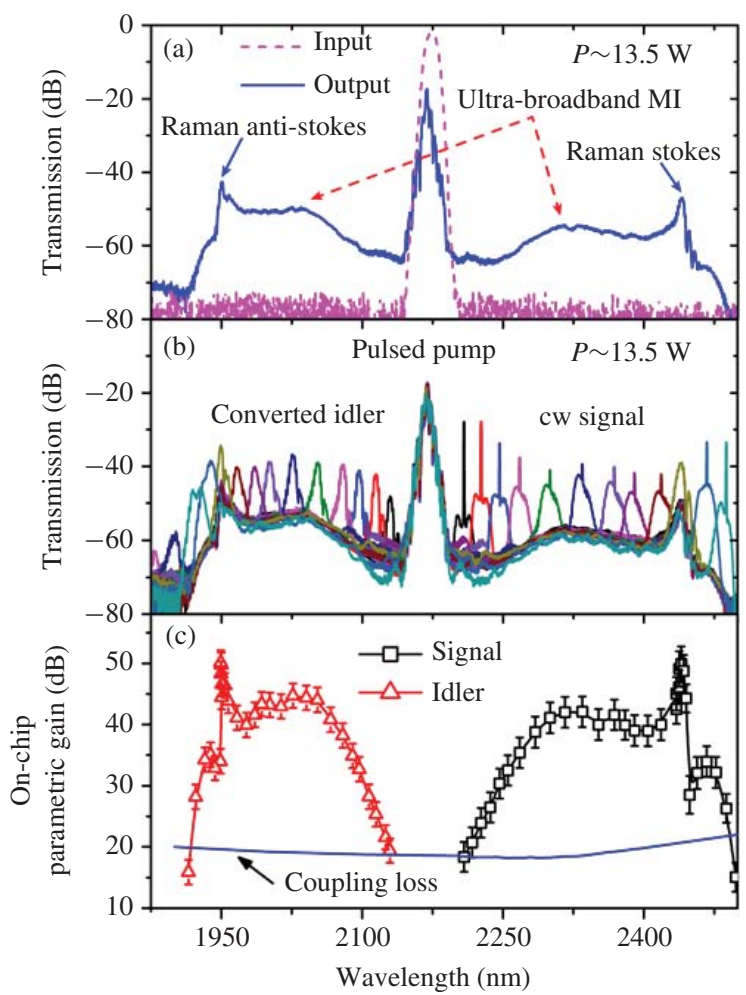

Fig. 5. (Color online) (a) Input (dashed magenta) and output (solid blue) pump spectrum, taken with an input peak power $\mathrm{P} 13.5 \mathrm{~W}$ and $\lambda=2173 \mathrm{~nm}$, illustrating ultra-broadband modulation instability. (b) Series of FWM spectra with the pulsed pump co-propagating with a cw mid-IR signal at various wavelengths. (c) Spectrum of on-chip parametric signal gain (black squares) and idler conversion gain (red triangles). Fiber-waveguide coupling loss is shown by the blue trace. The $3 \mathrm{~dB}$ gain bandwidth of the four-wave mixing process is about $100 \mathrm{~nm}$ around a wavelength of $2330 \mathrm{~nm}$.

levels within the same silicon waveguide [34]. In this experiment, the picosecond pump pulses were centered at a slightly shorter wavelength near $2120 \mathrm{~nm}$, as this pump wavelength was found to produce the broadest possible emission spectrum. The wavelength dependence of the extent of the supercontinuum is caused by the strong wavelength dependence of the second-order and fourth order dispersion terms as shown in Figure 4. The waveguide output spectra are shown in Figure 6 as the input coupled peak pump power is gradually increased from $3.1 \mathrm{~W}$ (green trace) to $12.7 \mathrm{~W}$ (black trace). As can be seen from this study, different nonlinear effects ultimately determine the shape and the extent of the generated supercontinuum. Even at the lowest input power, the pump pulse train's spectrum is broadened significantly by self phase modulation (SPM), as illustrated by the interference fringes appearing near $2120 \mathrm{~nm}$. In turn, this phenomenon causes associated broadening of the sidebands generated through modulation instability and Raman scattering. The broadband and the discrete phase matched side-bands are labeled as MI(1) and $\mathrm{MI}(2)$, respectively. Spontaneous Raman scattering can also clearly be observed, red-shifted by $15.6 \mathrm{THz}$ from the pump as expected. At a pump power of $7.9 \mathrm{~W}$, several new spectral components are observed, peaked near $1700 \mathrm{~nm}$ and $1600 \mathrm{~nm}$, respectively. The component at $1700 \mathrm{~nm}$ is generated through cascaded FWM, where the original MI(2) peak at 


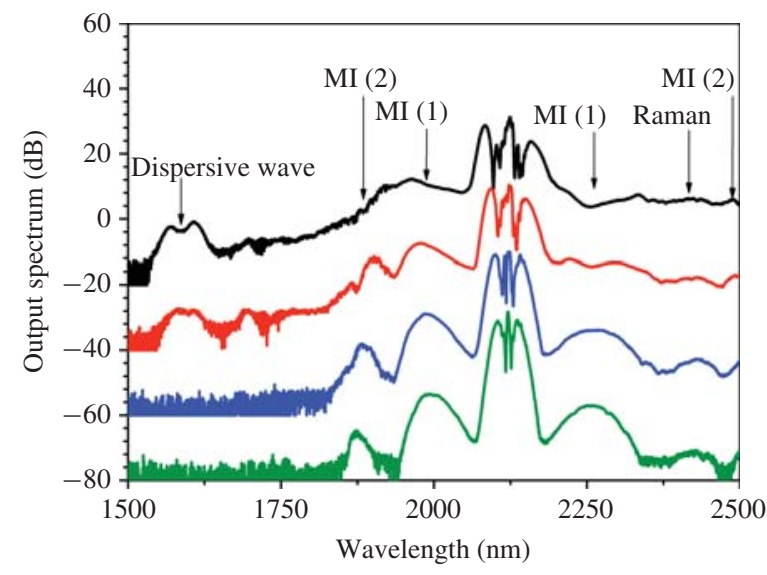

Fig. 6. Measured output spectrum for increasing values of coupled input peak power (color online) $3.1 \mathrm{~W}$ (green), $4.3 \mathrm{~W}$ (blue), $7.9 \mathrm{~W}$ (red), and $12.7 \mathrm{~W}$ (black). The spectra are vertically offset by multiples of $20 \mathrm{~dB}$ for clarity.

$1890 \mathrm{~nm}$ serves as the degenerate pump and the input pulse at $2120 \mathrm{~nm}$ acts as the signal. The peak around $1600 \mathrm{~nm}$ is believed to be the result of Cherenkov radiation, also referred to as dispersive wave generation [35]. Measurement of the spectral content of the supercontinuum using a Fourier Transform Infrared spectrometer (FTIR) showed a long-wavelength cut-off at $2.55 \mu \mathrm{m}$.

In contrast to previous supercontinua generated using planar waveguide circuits, where high-power femtosecond pulses were required [36], the short-wave infrared supercontinuum demonstrated here is generated from picosecond pulses with moderate peak powers. The combination of the relaxed pump pulse requirements and the large supercontinuum spectral range makes the silicon wire waveguide platform attractive for development of highly integrated white light infrared sources for spectroscopic applications. Moreover, the large concurrent parametric gain exhibited also allows realization of a broadly tunable optical parametric oscillator in the short-wave infrared [37].

\section{CONCLUSION}

In this paper we reported on our first steps in the field of long-wavelength silicon photonics. This research is fueled by the need for compact, integrated solutions for spectroscopic sensor systems in the MIR for biomedical applications and environmental monitoring, since many relevant molecules have "fingerprint" absorption lines within this wavelength range. The baseline for this research is the use of a CMOS compatible waveguide platform extended with III-V epitaxy for photodetectors. In a later stage integrated laser diodes will follow, which will rely on the bonding of a separate III-V die containing the laser epitaxy. The first successful demonstration of heterogeneously integrated short-wave infrared photodetectors on a silicon waveguide platform is reported, together with a comprehensive summary of the generation of short-wave infrared radiation through FWM in silicon photonic wires. These results show that there is a bright future for short-wave and mid-wave infrared photonic integrated circuits based upon a combination of silicon and III-V semiconductor photonic technology.

\section{REFERENCES}

[1] B. Jalali and S. Fathpour, "Silicon photonics," J. Lightw. Technol., vol. 24, no. 12, pp. 4600-4615, 2006.

[2] K. De Vos, I. Bartolozzi, E. Schacht, P. Bienstman, and R. Baets, "Silicon-on-insulator microring resonator for sensitive and label-free biosensing," Opt. Exp., vol. 15, no. 12, pp. 7610-7615, 2007.

[3] J. T. Robinson, L. Chen, and M. Lipson, "On-chip gas detection in silicon optical microcavities," Opt. Exp., vol. 16, no. 6, pp. 4296-4301, 2008.

[4] A. G. Yurtsever, P. Dumon, W. Bogaerts, and R. Baets, "Integrated photonic circuit in silicon on insulator for fourier domain optical coherence tomography," Proc. SPIE, vol. 75541B, pp. 75541-1-75541-5, Feb. 2010.

[5] J. G. Crowder, S. D. Smith, A. Vass, and J. Keddie, "Infrared methods for gas detection," in Mid-Infrared Semiconductor Optoelectronics. New York: Springer-Verlag, 2006.

[6] J. Brouckaert, G. Roelkens, S. Selvaraja, W. Bogaerts, P. Dumon, S Verstuyft, Z. Yu, D. Van Thourhout, and R. Baets, "Miniature integrated spectrometer fabricated on a silicon-on-insulator substrate," in Proc. IEEE Lasers Electro-Opt. Soc. 21st Annu. Meeting, Nov. 2008, pp. 5556.

[7] R. Soref, S. J. Emelett, and W. R. Buchwald, "Silicon waveguided components for the long-wave infrared region," J. Opt. A: Pure Appl. Opt., vol. 8, no. 10, p. 840, 2006.

[8] P. Y. Yang, G. Z. Mashanovich, I. Gomez-Morilla, W. R. Headley, G. T. Reed, E. J. Teo, D. J. Blackwood, M. B. H. Breese, and A. A. Bettiol, "Free-standing waveguides in silicon," Appl. Phys. Lett., vol. 90, no. 24, pp. 241109-1-241109-5, 2007.

[9] F. Li, D. S. Jackson, C. Grillet, E. Magi, D. Hudson, S. J. Madden, Y. Moghe, C. O'Brien, A. Read, S. G. Duvall, P. Atanackovic, B. J. Eggleton, and D. J. Moss, "Low propagation loss silicon-on-sapphire waveguides for the mid-infrared," Opt. Exp., vol. 19, no. 16, pp. 15212 15220, 2011.

[10] G. Z. Mashanovich, M. M. Miloševic, M. Nedeljkovic, N. Owens, B. Xiong, E. J. Teo, and Y. Hu, "Low loss silicon waveguides for the midinfrared," Opt. Exp., vol. 19, no. 8, pp. 7112-7119, 2011.

[11] T. Takeshita, T. Sato, M. Mitsuhara, Y. Kondo, and H. Oohashi, "Reliable $2.3 \mu \mathrm{m}$ wavelength highly strained InAs-InP MQW-DFB lasers with p/n-InP buried heterostructure," IEEE Photon. Technol. Lett., vol. 21, no. 13, pp. 896-898, Jul. 2009.

[12] J. Faist, T. Aellen, T. Gresch, M. Beck, and M. Giovannini, "Progress in quantum cascade lasers," Mid-Infrared Coher. Sour. Applicat., vol. 2, no. 1, pp. 171-192, 2008.

[13] A. Ducanchez, L. Cerutti, P. Grech, F. Genty, and E. Tournie, "Midinfrared GaSb-based EP-VCSEL emitting at $2.63 \mu \mathrm{m}$," Electron. Lett., vol. 45, no. 5, pp. 265-266, Feb. 2009.

[14] N. Hattasan, A. Gassenq, L. Cerutti, J. B. Rodriguez, E. Tournie, and G. Roelkens, "Heterogeneous integration of GaInAsSb p-i-n photodiodes on a silicon-on-insulator waveguide circuit," IEEE Photon. Technol. Lett., vol. 23, no. 23, pp. 1760-1762, Dec. 2011.

[15] G. Roelkens, J. Brouckaert, D. Van Thourhout, R. Baets, R. Notzel, and M. Smit, "Adhesive bonding of InP/InGaAsP dies to processed siliconon-insulator wafers using DVS-bis-benzocyclobutene," J. Electrochem. Soc., vol. 153, no. 12, pp. 1015-1019, 2006.

[16] [Online]. Available: http://www.ipgphotonics.com/Midirlasers.htm

[17] B. Kuyken, N. Hattasan, D. Vermeulen, S. K. Selvaraja, W. Bogaerts, W. Green, R. Baets, and G. Roelkens, "Highly efficient broadband siliconon-insulator grating couplers for the short wave infrared wavelength range," in Proc. Integrat. Photon. Res. Silicon Nano-Photon., Toronto, Canada, 2011, pp. 1-3.

[18] X. Liu, R. M. Osgood, Y. A. Vlasov, and W. M. J. Green, "Mid-infrared optical parametric amplifier using silicon nanophotonic waveguides," Nat. Photon., vol. 4, pp. 557-560, May 2010.

[19] S. Zlatanovic, J. S. Park, S. Moro, J. M. Chavez-Boggio, I. B. Divliansky, N. Alic, S. Mookherjea, and S. Radic, "Mid-infrared wavelength conversion in silicon waveguides using ultracompact telecom-band-derived pump source," Nat. Photon., vol. 4, no. 8, pp. 561-564, 2010.

[20] A. C. Turner-Foster, M. A. Foster, R. Salem, A. L. Gaeta, and M. Lipson, "Frequency conversion over two-thirds of an octave in silicon nanowaveguides," Opt. Exp., vol. 18, no. 3, pp. 1904-1908, 2010. 
[21] R. K. W. Lau, M. Menard, Y. Okawachi, M. A. Foster, A. C. TurnerFoster, R. Salem, M. Lipson, and A. L. Gaeta, "Continuous-wave midinfrared frequency conversion in silicon nanowaveguides," Opt. Lett., vol. 36, no. 7, pp. 1263-1265, 2011.

[22] J. I. Dadap, N. C. Panoiu, X. Chen, I.-W. Hsieh, X. Liu, C.-Y. Chou, E. Dulkeith, S. J. McNab, F. Xia, W. M. J. Green, L. Sekaric, Y. A. Vlasov, and R. M. Osgood, "Nonlinear-optical phase modification in dispersion-engineered Si photonic wires," Opt. Exp., vol. 16, no. 2, pp. 1280-1299, 2008.

[23] Q. Lin, O. J. Painter, and G. P. Agrawal, "Nonlinear optical phenomena in silicon waveguides: Modeling and applications," Opt. Exp., vol. 15, no. 25, pp. 16604-16644, 2007.

[24] R. Dekker, N. Usechak, M. Forst, and A. Driessen, "Ultrafast nonlinear all-optical processes in silicon-on-insulator waveguides," J. Phys. D: Appl. Phys., vol. 40, no. 14, pp. 249-271, 2007.

[25] O. Boyraz and B. Jalali, "Demonstration of a silicon Raman laser," Opt. Exp., vol. 12, no. 21, pp. 5269-5273, 2004.

[26] H. Fukuda, K. Yamada, T. Shoji, M. Takahashi, T. Tsuchizawa, T. Watanabe, J.-I. Takahashi, and S.-I. Itabashi, "Four-wave mixing in silicon wire waveguides," Opt. Exp., vol. 13, no. 12, pp. 4629-4637, 2005.

[27] Y.-H. Kuo, H. Rong, V. Sih, S. Xu, M. Paniccia, and O. Cohen, "Demonstration of wavelength conversion at $40 \mathrm{~Gb} / \mathrm{s}$ data rate in silicon waveguides," Opt. Exp., vol. 14, no. 24, pp. 11721-11726, 2006.

[28] M. A. Foster, A. C. Turner, J. E. Sharping, B. S. Schmidt, M. Lipson, and A. L. Gaeta, "Broad-band optical parametric gain on a silicon photonic chip," Nature, vol. 441, pp. 960-963, Jun. 2006.

[29] J. Leuthold, C. Koos, and W. Freude, "Nonlinear silicon photonics," Nat. Photon., vol. 4, no. 8, pp. 535-544, 2010.

[30] A. C. Turner, C. Manolatou, B. S. Schmidt, M. Lipson, M. A. Foster, J. E. Sharping, and A. L. Gaeta, "Tailored anomalous group-velocity dispersion in silicon channel waveguides," Opt. Exp., vol. 14, no. 10 pp. 4357-4362, 2006.

[31] E. Dulkeith, F. Xia, L. Schares, W. M. J. Green, and Y. A. Vlasov, "Group index and group velocity dispersion in silicon-on-insulator photonic wires," Opt. Exp., vol. 14, no. 9, pp. 3853-3863, 2006.

[32] A. D. Bristow, N. Rotenberg, and H. M. van Driel, "Two-photon absorption and Kerr coefficients of silicon for 850-2200 nm," Appl. Phys. Lett., vol. 90, no. 19, pp. 191104-191106, 2007.

[33] B. Kuyken, X. Liu, G. Roelkens, R. Baets, R. M. Osgood, and W. M. J. Green, "50 dB parametric on-chip gain in silicon photonic wires," Opt. Lett., vol. 36, no. 22, pp. 4401-4403, 2011.

[34] B. Kuyken, X. Liu, R. M. Osgood, R. Baets, G. Roelkens, and W. M J. Green, "Mid-infrared to telecom-band supercontinuum generation in highly nonlinear silicon-on-insulator wire waveguides," Opt. Exp., vol. 19, no. 21, pp. 20172-20181, 2011.

[35] N. Akhmediev and M. Karlsson "Cherenkov radiation emitted by solitons in optical fibers," Phys. Rev. A, vol. 51, no. 3, pp. 2602-2607, 1995.

[36] M. R. Lamont, B. Luther-Davies, D. Y. Choi, S. Madden, and B. J. Eggleton, "Supercontinuum generation in dispersion engineered highly nonlinear $(\mathrm{y}=10 / \mathrm{W} / \mathrm{m}) \mathrm{As}_{2} \mathrm{~S}_{3}$ chalcogenide planar waveguide," Opt. Exp., vol. 16, no. 19, pp. 14938-14944, 2008.

[37] B. Kuyken, X. Liu, R. M. Osgood, R. Baets, G. Roelkens, and W M. J. Green, "Widely tunable silicon mid-infrared optical parametric oscillator," in Proc. IEEE Group IV Photonics, 8th Int. Conf., London, U.K., Sep. 2011, pp. 338-340.

Gunther Roelkens received the Graduate degree (with highest honor) in electronics engineering (option: micro-electronics and opto-electronics) from Ghent University (UGent), Ghent, Belgium, and the Doctoral degree from the Photonics Research Group, Ghent University, in 2002 and 2007, respectively.

$\mathrm{He}$ has been with the Photonics Research Group, UGent, in the field of heterogeneous III-V/silicon photonics since 2002. In this paper, the technology for integrating III-V material on top of silicon-on-insulator waveguide circuits was developed and the integration of thin film III-V laser diodes and photodetectors on top of and coupled to the silicon-on-insulator waveguide circuit was demonstrated. He is currently a Tenure Track Professor with the same group. His current research interests include heterogeneous III-V/silicon integration, efficient fiber-chip coupling, all-optical signal processing, and mid-infrared photonic integrated circuits.
William M. J. Green (M'03) received the B.Sc. degree in engineering physics from the University of Alberta, Edmonton, AL, Canada, the M.Sc. degree in electrical engineering from the California Institute of Technology, Pasadena, and the Ph.D. degree in electrical engineering from the California Institute of Technology in 2005.

$\mathrm{He}$ is currently a Research Staff Member with IBM Thomas J. Watson Research Center, Yorktown Heights, NY. His current research interests include the design and systems-level characterization of integrated electrooptic devices for low-power, high-speed optical modulation and switching within on-chip optical networks, study of nonlinear silicon waveguide devices and circuits for all-optical parametric signal processing, and broadband midinfrared light source generation.

Dr. Green is a member of the IEEE Photonics Society and the Optical Society of America.

Bart Kuyken received the B.S. degree in engineering and physics from Ghent University (UGent), Ghent, Belgium, in 2006 and 2008, respectively, the M.Sc. degree in electrical engineering (option: electronic circuits and systems) from Ghent University, and another M.Sc. degree in electrical engineering from Stanford University, Stanford, CA, in 2008 and 2009, respectively.

He has been with the Photonics Research Group, UGent, on a doctoral fellowship from the Flemish Research Foundation since 2009.

Xiaoping Liu received the Ph.D. degree from the Department of Electrical Engineering, Columbia University, New York, NY, in 2011. His Ph.D. work involved a study of constructing silicon-based photonic integrated circuits, characterizing various ultrafast nonlinear effects in silicon nanophotonic wires, and investigating mid-infrared parametric amplification in silicon wires.

$\mathrm{He}$ is currently a Research Member with OFS Laboratories, Somerset, NJ. His current research interests include novel fiber optic components for high power lasers, sensing networks, and bio-medical applications.

Nannicha Hattasan received the B.E. degree in electronics engineering from the King Mongkut's Institute of Technology Ladkrabang, Bangkok, Thailand, the M.Eng.Sc. degree in microelectronics from the University of New South Wales, Sydney, Australia, in 2004, the M.Sc. degree in photonics from the Royal Institute of Technology, Stockholm, Sweden, and the M.Sc. degree from Ghent University, Ghent, Belgium, in 2009. She is currently pursuing the $\mathrm{Ph} . \mathrm{D}$. degree in heterogeneous integration of III-V and silicon-on-insulator.

Her current research interests include Si photonics, III-V diode lasers, photodiodes, hybrid integration, and technology development.

Alban Gassenq was born in Montpellier, France, in August 1983. He received the Masters degree in optoelectronics and electronics from Université Montpellier 2, Montpellier, and the Ph.D. degree in optoelectronics materials from Nanomir Group, Institut d'Electronique du Sud, Orsay, France, in 2007 and 2010, respectively.

He is currently a Post-Doctoral Fellow with the Photonics Research Group, Ghent University, Ghent, Belgium. His current research interests include the design and development of sources and photodetectors emitting in 2-5 $\mu \mathrm{m}$ range.

Laurent Cerutti received the Ph.D. degree from Université Montpellier 2, Montpellier, France, in 2004, which dealt with the molecular beam epitaxy of Sb-based vertical-cavity surface-emitting lasers for the mid-infrared wavelength range.

He is currently an Associate Professor with Université Montpellier 2. From 2004 to 2006, he was a Post-Doctoral Fellow with Instituto de Sistemas Optoelectrónica y Microtecnología, Universidad Politécnica, Madrid, Spain, working on the molecular beam epitaxy and studies of III-N nanocolumns for generation of white light and bio-photonics. In 2006, he joined the Mid-IR Group of Institut d'Electronique du Sud, Université Montpellier 2. He is the author or co-author of approximately 30 papers in refereed journals. 
Jean Baptiste Rodriguez received the Ph.D. degree from Université Montpellier 2, Montpellier, France, in 2005, for his work on InAs/GaSb superlattices grown by molecular beam epitaxy for room-temperature detection in the 3-5 $\mu \mathrm{m}$ wavelength range.

He was a Post-Doctoral Fellow with the Center for High Technology Material, University of New-Mexico, Albuquerque, from 2005 to 2007 , working on the design, fabrication, and characterization of InAs/GaSb-based infrared focal plane arrays. In 2007, he joined the Mid-IR Group of Institut d'Electronique du Sud, Université Montpellier 2, where he has been holding a Centre National de la Recherche Scientifique (CNRS) position since 2008. He is Chargé de Recherche in CNRS. He is author or co-author of approximately 30 papers in refereed journals.

Richard M. Osgood (SM'82-F'87) received the B.Sc. degree from U.S. Military Academy, West Point, NY, the M.S. degree from Ohio State University, Columbus, and the Ph.D. degree from the Massachusetts Institute of Technology, Cambridge.

$\mathrm{He}$ is currently a Higgins Professor of applied physics and electrical engineering with Columbia University, New York, NY. His current research interests include optical, chemical physics, electrical engineering, development of new infrared and ultraviolet lasers, and different applications of laserinduced chemistry.

Dr. Osgood is a member of the American Chemical Society and the Materials Research Society and is a fellow of the Optical Society of America and the American Physical Society.

Eric Tournié was born in November 1962. He received the Ph.D. degree from Université Montpellier 2 (UM2), Montpellier, France.

$\mathrm{He}$ was a Staff Member of the MBE Groups, Max-Planck-Institute, Stuttgart, Germany, and Paul-Drude-Institute, Berlin, Germany, working on InAs-based highly strained heterostructures on InP and GaAs substrates from 1990 to 1993. In 1993, he joined Center for Research on Heteroepitaxy and its Applications/Centre National de la Recherche Scientifique (CNRS), Valbonne, Germany, where he worked on ZnSe-based heterostructures for blue-green lasers. In 1999, he initiated a program on GaInNAs heterostructures and devices for telecom and solar-cells applications. In 2002, he became a Professor of electrical engineering UM2, where he is currently the Head of the Mid-IR Group, Institut d'Electronique du Sud, UMR CNRS. He coordinated the GINA1.5 FET-IST (FP5) Project and the DOMINO Project (FP6) from 2005 to 2008 and coordinates the MIRNANO Project (ANR PNANO 2008).
He has been the Principal Investigator for UM2 in the NEMIS Project (FP6, 2006-2009) and the TRAIN2 Project (FEDER SUDOE 2011-2012). He is the author/co-author of 160 papers in refereed journals and contributed 80 communications in international conferences.

Dr. Tournié is the Program Committee Member and/or International Advisory Committee Member of the International Conference on Mid-Infrared Optoelectronics: Materials and Devices, the International on Molecular Beam Epitaxy Conference, and the International Symposium on Compounds Semiconductors Series. He serves regularly as an expert for French programs as well as the Engineering and Physical Sciences Research Council, U.K., and the IST priority of FP6 and FP7, and a referee for several scientific journals. Since 2010, he has been an Editorial Board Member of the Journal of Physics D: Applied Physics.

Roel G. Baets (F'07) received the M.Sc. degree in electrical engineering from Ghent University (UGent), Ghent, Belgium, another M.Sc. degree from Stanford University, Stanford, CA, and the Ph.D. degree from UGent, in 1980, 1981, and 1984, respectively.

He is a Full Professor with UGent. He is associated with Illinois Manufacturing Extension Center (IMEC), Peoria. He has management responsibilities within the Photonics Research Group, UGent, the Center for Nano and Biophotonics (NB Photonics), UGent, the International Erasmus Mundus M.Sc. Program in Photonics, and the joint UGent-IMEC Research Program on Silicon Photonics. From 1984 to 1989, he held a post-doctoral position at IMEC (with detachment to UGent). Since 1989, he has been a Professor with the Engineering Faculty, UGent, where he founded the Photonics Research Group. From 1990 to 1994, he was a part-time Professor with Technical University, Delft, The Netherlands. From 2004 to 2008, he was with the Technical University of Eindhoven, Eindhoven, The Netherlands, where he has mainly worked in the field of integrated photonic components and has made contributions to research on semiconductor laser diodes, guided wave and grating devices, and the design and fabrication of photonic integrated circuits, both in III-V semiconductors and silicon. As part of a team of six professors, he leads the Photonics Research Group, UGent. With about 60 researchers, this group is involved in numerous national and international research programs. The silicon photonics activities of the group are part of a joint research initiative with IMEC. $\mathrm{He}$ is the Director of the Multidisciplinary Center for NB Photonics, UGent, founded in 2010. He was a Co-Founder of the interuniversity UGent-VUB M.Sc. Program in Photonics and the International Erasmus Mundus M.Sc. Program in Photonics of which he chairs the Board.

Dr. Baets is a grant holder of the Methusalem Program of the Flemish Government and the European Research Council. 